\title{
Impacts of Smart Waters on Calcite-Oil Interactions Quantified by "soft tip" Atomic Force Microscopy (AFM) and Surface Complexation Modeling (SCM)
}

\section{Supporting Information 2}

Hongna Ding a,d, Srinivas Mettu *b,c, Sheikh S Rahman*,d

a School of Petroleum Engineering, Northeast Petroleum University, Daqing, Heilongjiang Province, China.

${ }^{b}$ School of Chemistry and the Department of Chemical Engineering, University of Melbourne, Parkville, Victoria 3010, Australia.

${ }^{\mathrm{c}}$ Chemical and Environmental Engineering, RMIT University, Melbourne, Victoria 3000, Australia.

${ }^{d}$ School of Minerals and Energy Resources Engineering, University of New South Wales, Sydney, NSW 2052, Australia.

Corresponding Authors: srinivas.mettu@unimelb.edu.au; sheik.rahman@unsw.edu.au

\section{XRD analysis result of Iceland spar}

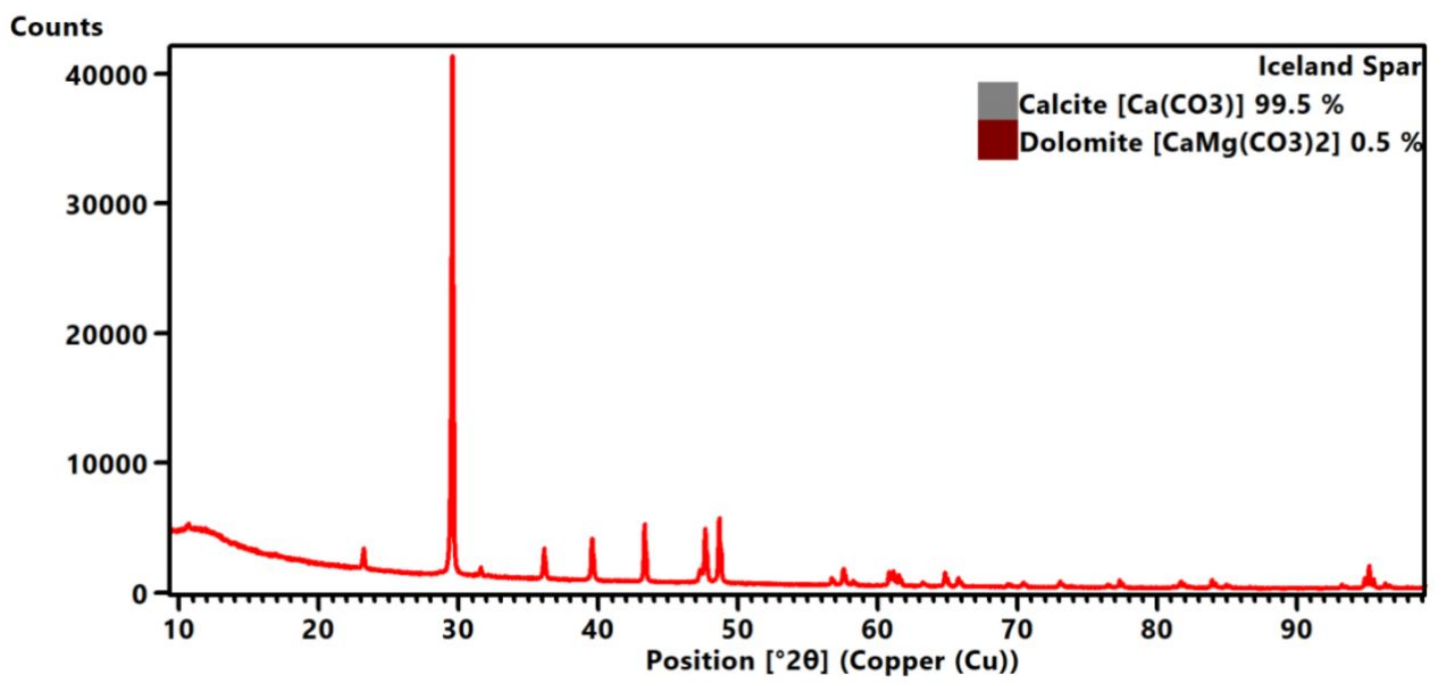

Figure S1. X-ray diffraction spectrum and processed plot of Iceland spar

\section{Influence of hydrodynamic force}

The hydrodynamic force is often measured at high velocities of AFM piezo ${ }^{1-4}$. In Dagastine et al.'s study, the authors tracked the force behaviors of two decane droplets in $1 \mathrm{mM}$ $\mathrm{NaNO}_{3}$ and $10 \mathrm{mM}$ SDS solution as a function of piezo velocities. They found that the two force curves at $v=80$ and $120 \mathrm{~nm} / \mathrm{s}$ superimposed on each other, indicating no influences of hydrodynamic effects. On the contrary, the hydrodynamic force started to become pronounced at 
$v>500 \mathrm{~nm} / \mathrm{s}$, which introduced strong repulsions to the oil-oil interactions, causing an increase in the force magnitude with the velocity ${ }^{1}$. Thus, it is normally preferred to use relatively low piezo velocities to measure the equilibrium forces. It has been reported that the hydrodynamic force is often generated owing to a quick drainage of the thin water film between the tip and the sample surface ${ }^{5}$.

We had measured the forces between a droplet of mineral oil (hydrocarbons and aromatics) and calcite surface in $0.11 \mathrm{M} \mathrm{CaCl}_{2}$ solution at velocity of $100-5000 \mathrm{~nm} / \mathrm{s}$. As shown in Figure $\mathrm{S} 2$, the hydrodynamic force was not significant at $v \leq 1000 \mathrm{~nm} / \mathrm{s}$ because the attractions between the oil droplet and the calcite surface is too large to be conquered by the repulsive hydrodynamic force. However, the hydrodynamic force becomes significant at $v=5000 \mathrm{~nm} / \mathrm{s}$ (see Figure S2), which changed the attractive interactions of mineral oil with calcite surface into repulsive, and moreover, caused the approach and retract curve to be separated when the oil droplet (soft tip) was fully released from the calcite surface due to energy dissipation.

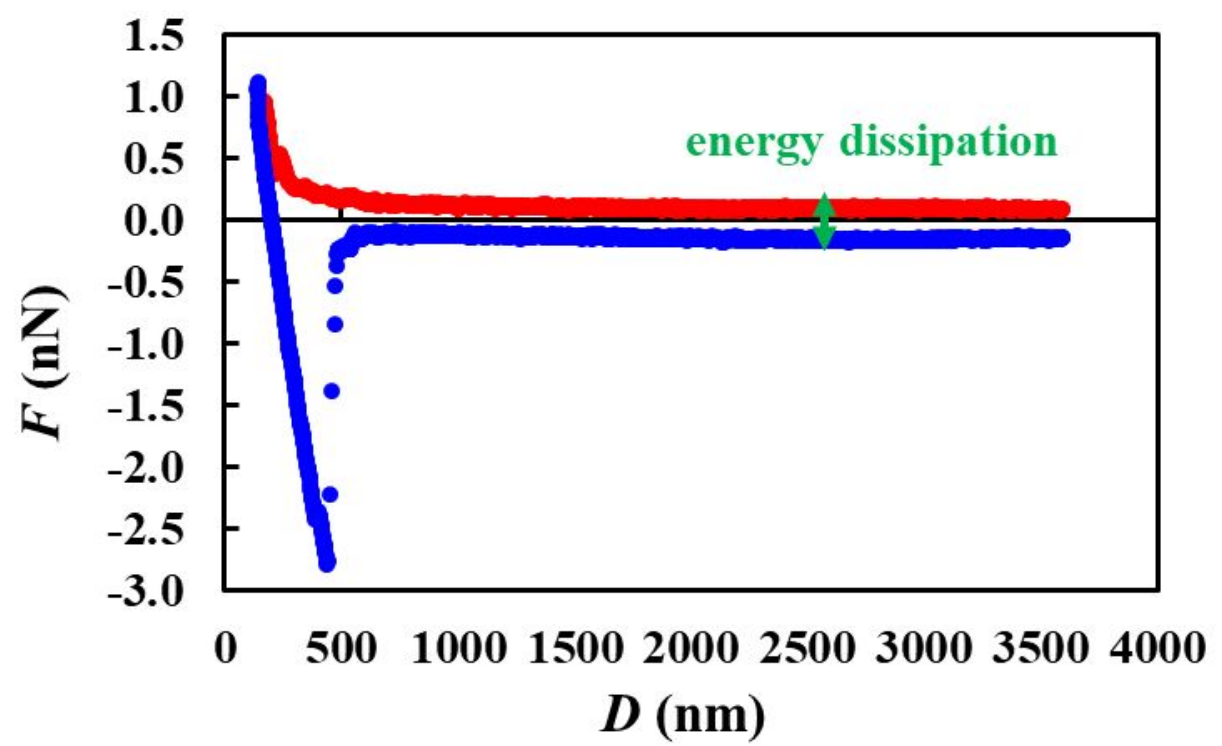

Figure S2. Force-distance curve of calcite surface and mineral oil in $0.11 \mathrm{M} \mathrm{CaCl}_{2}$ solution at $v=5000 \mathrm{~nm} / \mathrm{s}$ and $\mathrm{TP}=1000 \mathrm{pN}$. 
3. Adhesions in SWSO solution

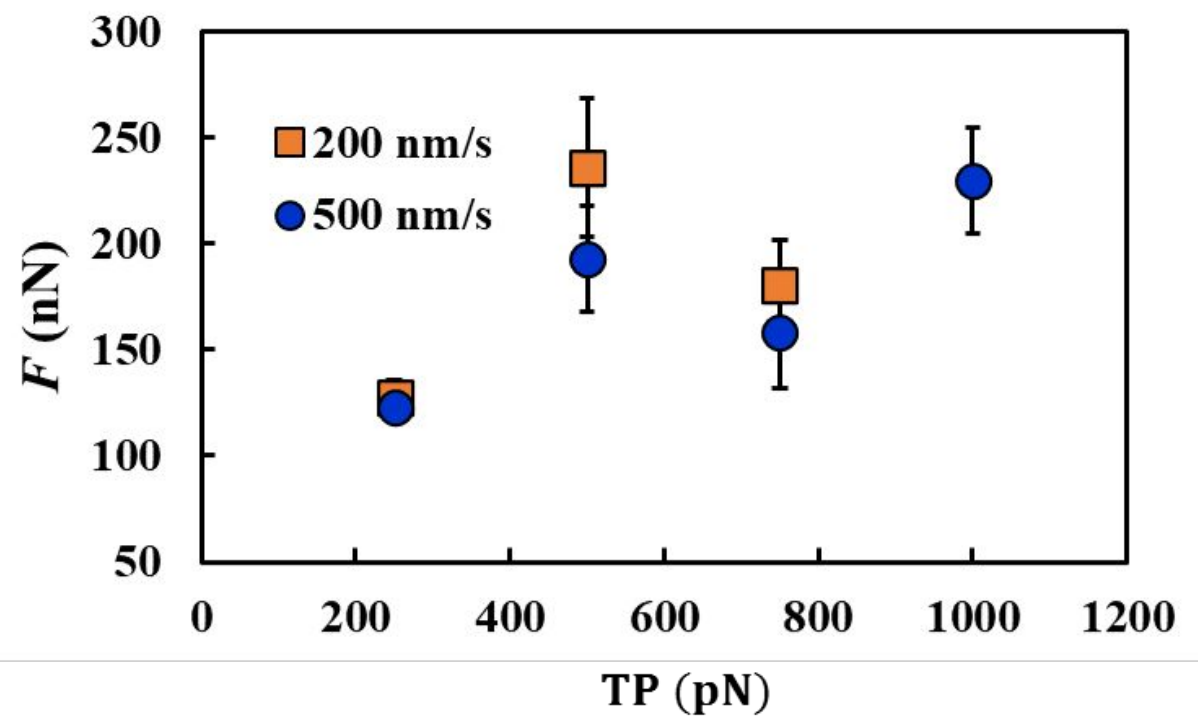

Figure S3. Adhesions of calcite surface and crude oil in SWSO solution with TP=250-1000 pN at $v=200 \mathrm{~nm} / \mathrm{s}$ (orange cubes) and $v=500 \mathrm{~nm} / \mathrm{s}$ (blue dots). The error bars show the $95 \%$ confidence of 5-9 measurements.

4. SCM results of $\mathrm{pH}$ and IS

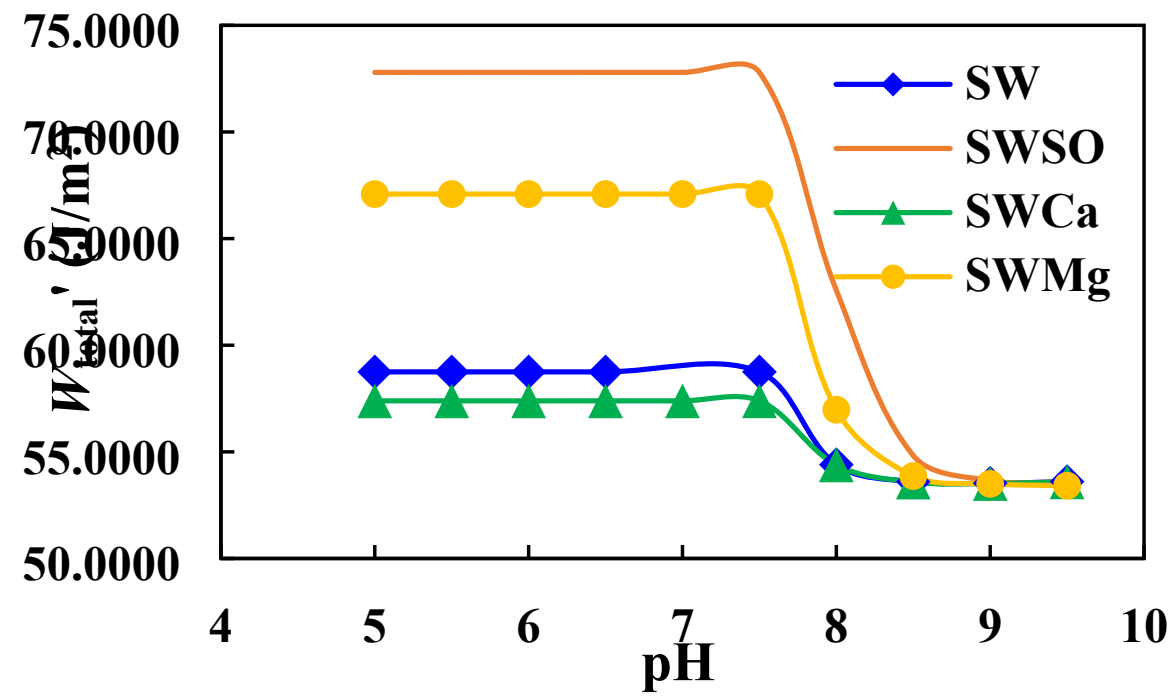

Figure S4. Adhesion energy of crude oil with calcite surface in four smart waters as a function of solution $\mathrm{pH}$. 
Table S1 Surface complexes and adhesion energy at $\mathrm{pH}=8$ and/or Ionic Strength $(I S)=2.3 \mathrm{M}$

\begin{tabular}{|c|c|c|c|c|}
\hline original & $\begin{array}{c}>\mathrm{CaOH}_{2}^{+}\left(-\mathrm{COO}^{-}\right) \\
(\text {mole })\end{array}$ & $\begin{array}{c}>\mathrm{CO}_{3} \mathrm{Ca}^{+}\left(-\mathrm{COO}^{-}\right) \\
(\text {mole })\end{array}$ & $\begin{array}{c}>\mathrm{CO}_{3} \mathrm{Mg}^{+}\left(-\mathrm{COO}^{-}\right) \\
\text {(mole })\end{array}$ & $\begin{array}{c}W_{\text {total }} \\
\left(\mathrm{mJ} / \mathrm{m}^{2}\right)\end{array}$ \\
\hline SW & $7.10 \times 10^{-22}$ & $7.83 \times 10^{-10}$ & $7.52 \times 10^{-9}$ & 56.002 \\
\hline SWSO & $6.45 \times 10^{-22}$ & $3.86 \times 10^{-9}$ & $4.44 \times 10^{-9}$ & 65.927 \\
\hline SWCa & $4.35 \times 10^{-22}$ & $2.44 \times 10^{-10}$ & $8.06 \times 10^{-9}$ & 54.267 \\
\hline SWMg & $7.53 \times 10^{-22}$ & $2.49 \times 10^{-9}$ & $5.81 \times 10^{-9}$ & 61.508 \\
\hline$I S=2.3 \mathrm{M}$ & $\begin{array}{c}>\mathrm{CaOH}_{2}^{+}\left(-\mathrm{COO}^{-}\right) \\
(\text {mole })\end{array}$ & $\begin{array}{c}>\mathrm{CO}_{3} \mathrm{Ca}^{+}\left(-\mathrm{COO}^{-}\right) \\
(\text {mole })\end{array}$ & $\begin{array}{c}>\mathrm{CO}_{3} \mathrm{Mg}^{+}\left(-\mathrm{COO}^{-}\right) \\
(\text {mole })\end{array}$ & $\begin{array}{c}\begin{array}{c}W_{\text {total }} \\
\left(\mathrm{mJ} / \mathrm{m}^{2}\right)\end{array} \\
\end{array}$ \\
\hline SW & $7.10 \times 10^{-22}$ & $7.83 \times 10^{-10}$ & $7.52 \times 10^{-9}$ & 56.002 \\
\hline SWSO & $6.45 \times 10^{-22}$ & $3.86 \times 10^{-9}$ & $4.44 \times 10^{-9}$ & 65.667 \\
\hline SWCa & $4.35 \times 10^{-22}$ & $2.44 \times 10^{-10}$ & $8.06 \times 10^{-9}$ & 54.262 \\
\hline SWMg & $7.53 \times 10^{-22}$ & $2.49 \times 10^{-9}$ & $5.81 \times 10^{-9}$ & 61.338 \\
\hline $\mathrm{pH}=8$ & $\begin{array}{c}>\mathrm{CaOH}_{2}^{+}\left(-\mathrm{COO}^{-}\right) \\
(\text {mole }) \\
\end{array}$ & $\begin{array}{c}>\mathrm{CO}_{3} \mathrm{Ca}^{+}\left(-\mathrm{COO}^{-}\right) \\
(\mathrm{mole})\end{array}$ & $\begin{array}{c}>\mathrm{CO}_{3} \mathrm{Mg}^{+}\left(-\mathrm{COO}^{-}\right) \\
(\text {mole })\end{array}$ & $\begin{array}{c}W_{\text {total }} \\
\left(\mathrm{mJ} / \mathrm{m}^{2}\right)\end{array}$ \\
\hline SW & $4.62 \times 10^{-22}$ & $2.81 \times 10^{-10}$ & $8.02 \times 10^{-9}$ & 54.386 \\
\hline SWSO & $5.31 \times 10^{-22}$ & $2.82 \times 10^{-9}$ & $5.48 \times 10^{-9}$ & 62.571 \\
\hline SWCa & $4.62 \times 10^{-22}$ & $2.82 \times 10^{-10}$ & $8.02 \times 10^{-9}$ & 54.385 \\
\hline SWMg & $5.08 \times 10^{-22}$ & $1.09 \times 10^{-9}$ & $7.21 \times 10^{-9}$ & 57.002 \\
\hline $\begin{array}{c}\mathrm{pH}=8, \\
I S=2.3 \mathrm{M}\end{array}$ & $\begin{array}{c}>\mathrm{CaOH}_{2}^{+}\left(-\mathrm{COO}^{-}\right) \\
(\text {mole })\end{array}$ & $\begin{array}{c}>\mathrm{CO}_{3} \mathrm{Ca}^{+}\left(-\mathrm{COO}^{-}\right) \\
(\text {mole })\end{array}$ & $\begin{array}{c}>\mathrm{CO}_{3} \mathrm{Mg}^{+}\left(-\mathrm{COO}^{-}\right) \\
(\text {mole })\end{array}$ & $\begin{array}{c}\begin{array}{c}W_{\text {total }} \\
\left(\mathrm{mJ} / \mathrm{m}^{2}\right)\end{array} \\
\end{array}$ \\
\hline SW & $4.63 \times 10^{-22}$ & $2.80 \times 10^{-10}$ & $8.02 \times 10^{-9}$ & 54.379 \\
\hline SWSO & $5.48 \times 10^{-22}$ & $2.65 \times 10^{-9}$ & $5.66 \times 10^{-9}$ & 61.998 \\
\hline SWCa & $4.63 \times 10^{-22}$ & $2.79 \times 10^{-10}$ & $8.02 \times 10^{-9}$ & 54.379 \\
\hline SWMg & $5.21 \times 10^{-22}$ & $1.03 \times 10^{-9}$ & $7.28 \times 10^{-9}$ & 56.780 \\
\hline
\end{tabular}

\section{References}

1. Dagastine, R. R.; Stevens, G. W.; Chan, D.; Grieser, F., Forces between two oil drops in aqueous solution measured by AFM. Journal of colloid and interface science 2004, 273 (1), 339342.

2. $\quad$ Carnie, S. L.; Chan, D. Y.; Lewis, C.; Manica, R.; Dagastine, R. R., Measurement of dynamical forces between deformable drops using the atomic force microscope. I. Theory. Langmuir 2005, 21 (7), 2912-2922.

3. Vakarelski, I. U.; Higashitani, K., Dynamic features of short-range interaction force and adhesion in solutions. Journal of colloid and interface science 2001, 242 (1), 110-120.

4. $\quad$ Mettu, S.; Berry, J. D.; Dagastine, R. R., Charge and Film Drainage of Colliding Oil Drops Coated with the Nonionic Surfactant C12E5. Langmuir 2017, 33 (20), 4913-4923. 5. Bonaccurso, E.; Kappl, M.; Butt, H.-J., Thin liquid films studied by atomic force microscopy. Current Opinion in Colloid \& Interface Science 2008, 13 (3), 107-119. 